SECTION 4. Computer science, computer engineering and automation.

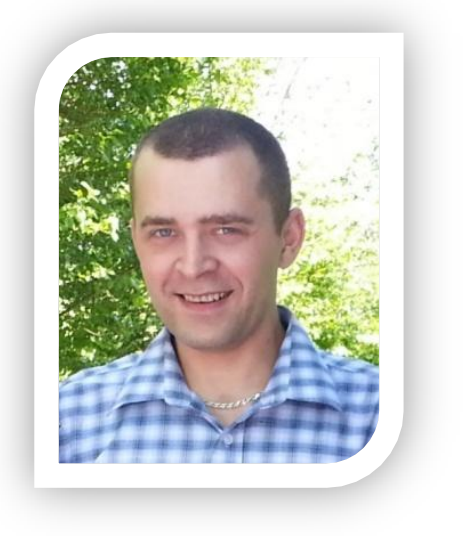

Shyrynkhanova Dinara Zhaksylykovna

1 year magistr of the speciality "Information systems "

Taraz State University named after M.Kh. Dulati, Kazakhstan
Shevtsov Alexandr Nikolayevich candidate of technical Sciences, associate Professor of the Department «Applied mathematics» Taraz State University named after M.Kh. Dulati, Kazakhstan

\title{
DEVELOPMENT OF ALGORITHMS AND APPLICATION COMPONENT MODEL TO ANALYZE AND FIX ERRORS TEST.
}

The process of working of the Center for testing University, and includes the reception of test tasks of teachers in various disciplines, validation of recruitment and clearance for entry into the database and its further use in examinations.

One of the most difficult and crucial moments - is checking the correctness of test and identification of errors. This issue is devoted to this article.

Keywords: test automation, Delphi.

\section{РАЗРАБОТКА АЛГОРИТМОВ И ПРИЛОЖЕНИЯ КОМПОНЕНТНОЙ МОДЕЛИ ДЛЯ АНАЛИЗА И ИСПРАВЛЕНИЯ ОШИБОК ЭКЗАМЕНАЦИОННОГО ТЕСТА}

- Процесс работы Центра тестирования университета, включает в себя - прием тестовых заданий преподавателей по различным дисциплинам, проверку правильности набора и оформления, занесение в базу данных и дальнейшее его использование на экзаменах. 
Одним из наиболее сложных и ответственных моментов - является проверка правильности оформления тестового задания, и выявление допущенных ошибок. Именно этой проблеме посвящена данная статья

Ключевые слова: экзамен, тест, автоматизачия, Дельфи.

Разработаем программу, которая в сочетании с набором уже используемых в Центре тестирования, будет образовывать компонентную модель. А также алгоритмы для автоматической проверки теста, нахождения ошибок, и автоматического их исправления в соответствии с требованиями.

Определим правильную форму тестового задания (рис.1-2).

1. Квадратная 'матрица нназывается диагональной, если.....

А) все'элементы вне 'главной диагонали равны 'нулю-

В) все-элементы побочной-диагонали равны нулю-

C) все·элементы главной диагонали равны'нулюๆ

D)·все·элементы матрицы равны 'нулю-

Е)·все·элементы·вне'главной·диагонали равны·единице

-

2. Прямоугольная 'матрица $\cdot A$ размеров $m \times n$ называется квадратной, если $\cdot \ldots$ ๆ

A) $\cdot m=n \cdot$

B) $\cdot m \neq n$ व

Рисунок 1 - Образец тестового задания.

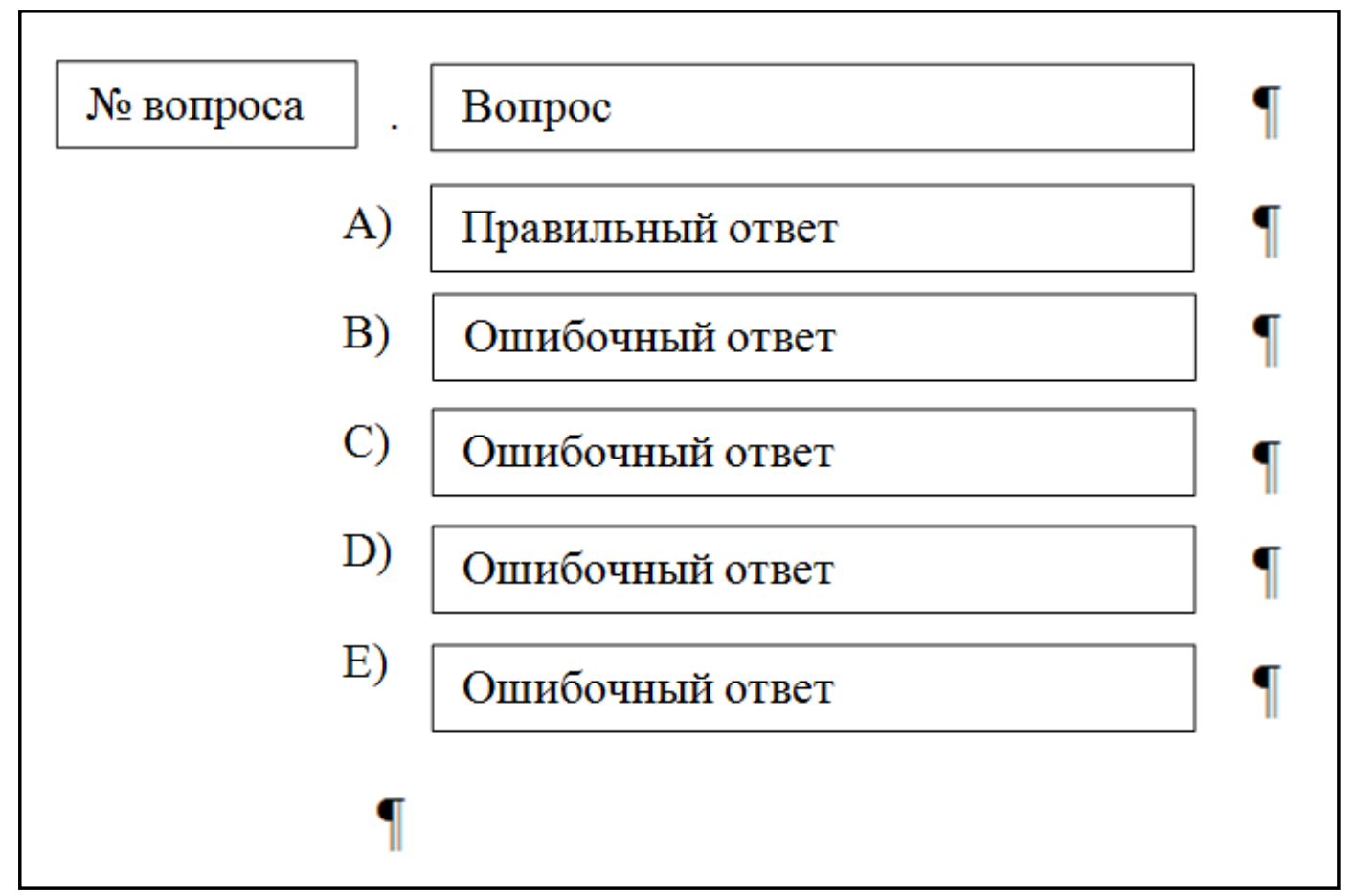

Рисунок 2 - Блок схема тестового задания. 
Определим наиболее типичные ошибки встречающиеся в тестовых заданиях:

- Наличие лишнего знака q (Enter), два и более подряд.

- Знак 9 (Enter) находится между вариантами ответов.

- Два правильных ответа.

- Ошибочная нумерация вопросов.

- Отсутствие точки после номера вопроса.

- Отсутствие скобки после буквы(индекса) ответа.

- Два одинаковых индекса ответов.

- Вопрос разбит на две строки знаком - (Enter)

- Лишние пробелы в зоне индексов вопросов и ответов.

- Повторяющиеся вопросы в тесте.

Разработаем программу в среде «Embarcadero® RAD Studio 2010». Редактирование теста будем осуществлять непосредственно в программе Microsoft Word, посылая определенные запросы на его сервер. Для этого встроим основные серверные компоненты Word в нашу программу. Нам потребуются:

- MSWORD.OLB

- Office_Tlb.dcu

- Office_TLB.pas

- VBIDE_TLB.dcu

- VBIDE_TLB.pas

- Word_tlb.dcu

- Word_TLB.pas

Теперь займемся разработкой алгоритмов.

Для нахождения лишних знаков ๆ (Enter), втречающихся в тесте, будем проходить по всему тесту сравнивая две рядом стоящих строки и в случае их равентства будем удалять вторую из них (проверяя наличие постого переноса строки).

procedure TForm1.Button2Click(Sender: TObject);

Var

sss, sss1:String;

$\mathrm{N}, \mathrm{I}, \mathrm{j}$ : Integer;

v, vk: OleVariant;

begin

form1.Left:=10; form1.Top:=10;

memo1.Clear;

WordApp.Options.CheckSpellingAsYouType:=False; 


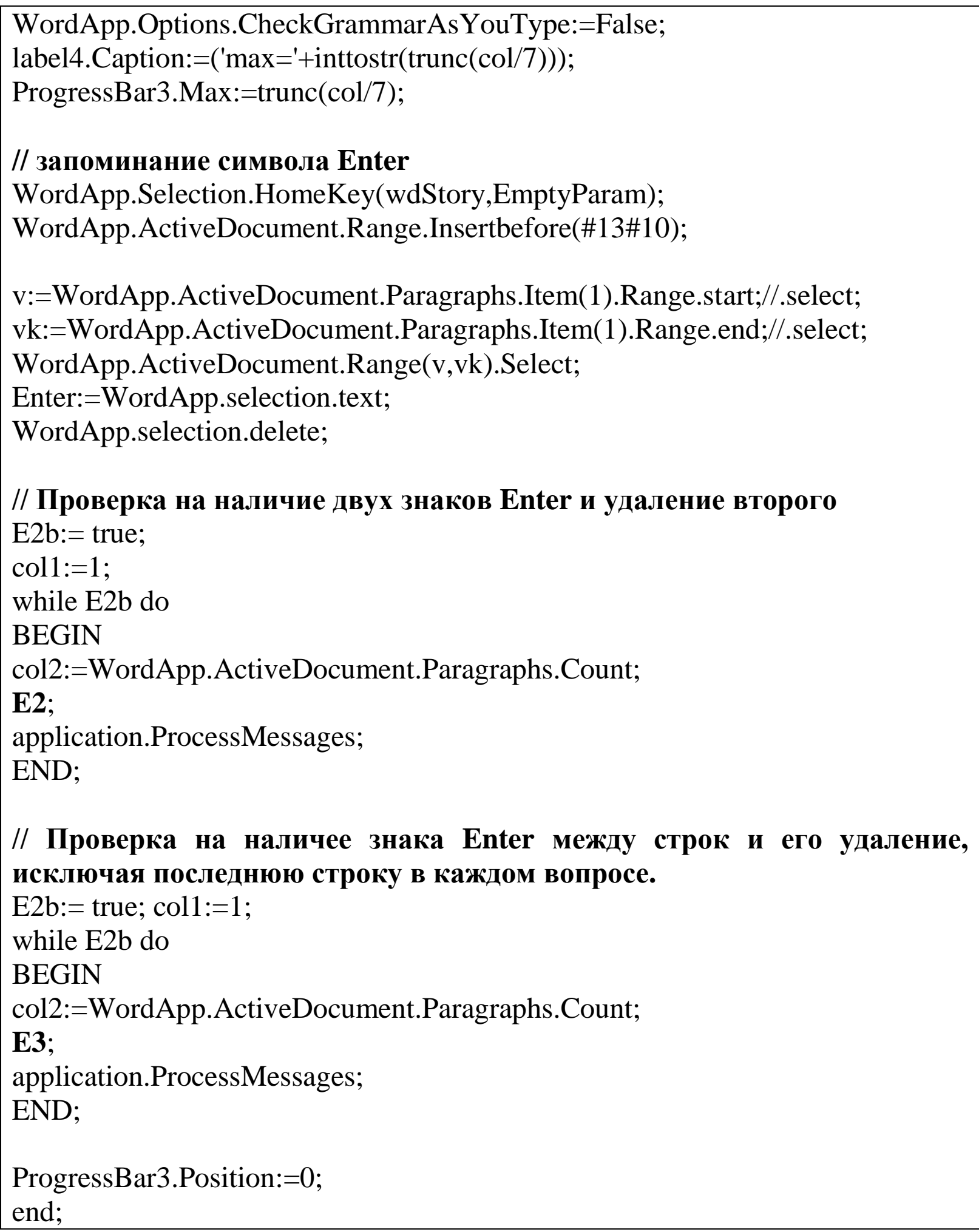

Здесь вводятся дополнительно две подпрограммы $\mathbf{E 2}$ и $\mathbf{E 3}$, которые как раз и обрабатывают команды для сервера, находят и исправляют отмеченные ошибки. Рассмотрим их более подробно:

code:

procedure TForm1.E2; // два Enter 


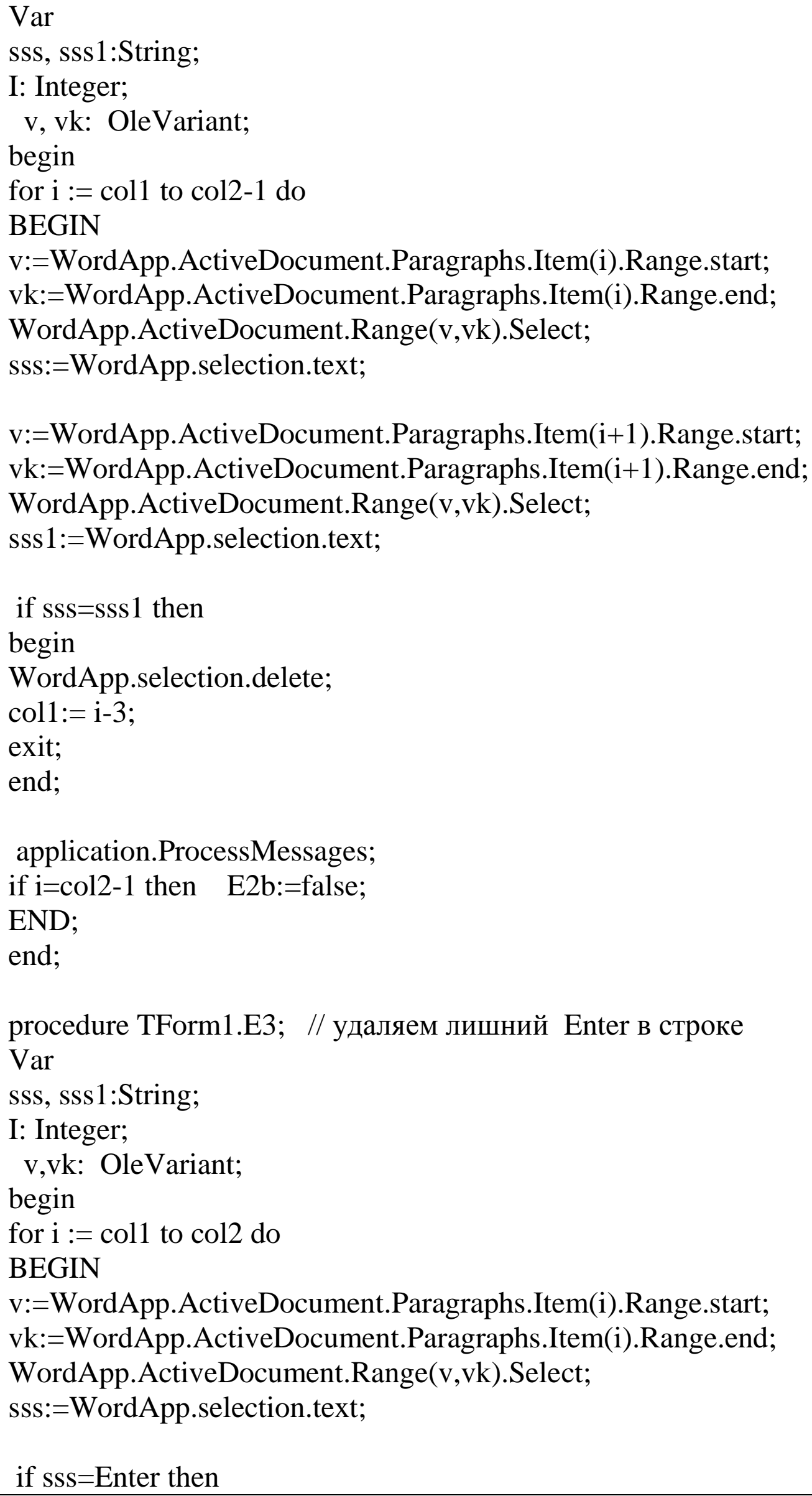




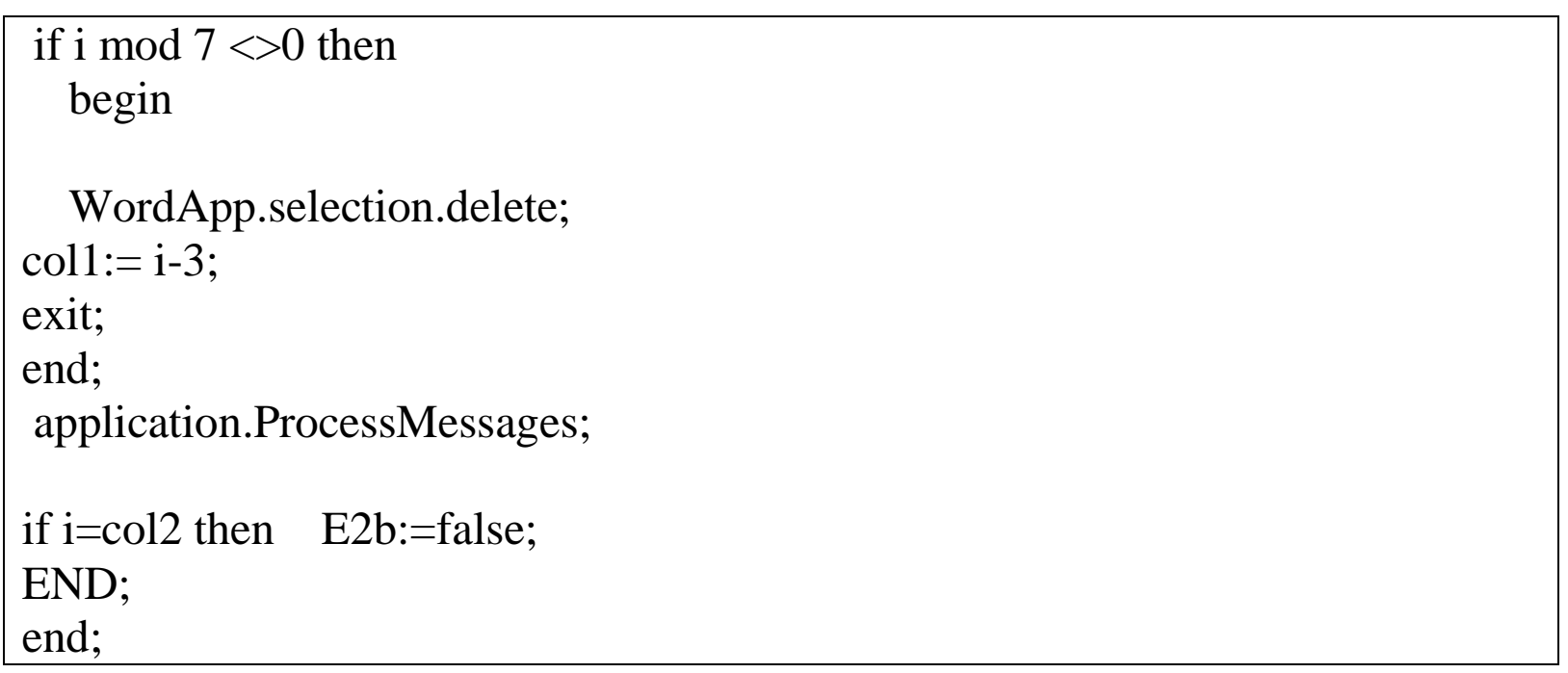

Получим следующую программу (рис.3) работующую на основе OLE технологии и реализующую компонентную модель. Загрузка данных из файла производится посредством Drag\&Drop.

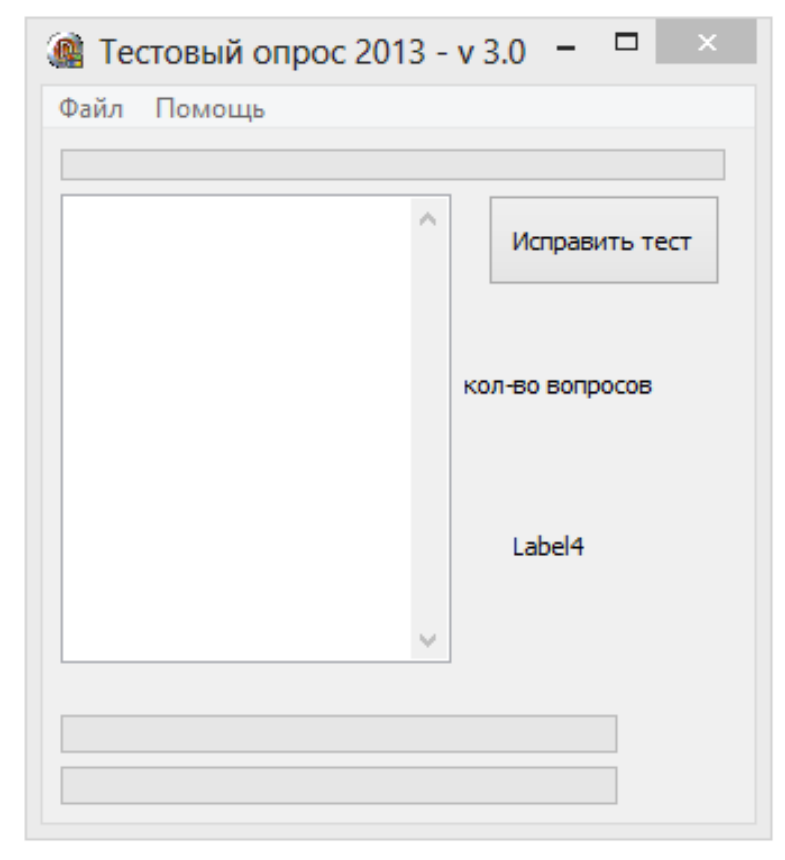

\section{Рисунок 3 - Программа.}

Проверим ее функциональность на тесте (рис. 4-5), как видим после автоматического исправления - тест соответствует требованиям. Разработанная программа исправляет пропущенные переносы на другую строку, а также удаляет лишние. 
1. Квадратная матрица называется ддиагональной, если.....

А) все-элементы внетлавной диагонали равны нулю-

-

В) все-элементы побочной диагонали равны нулюণ

C) все-элементы тлавной диагонали равны нулюণ

ฯ

D)все-элементы матрицы равны нулюף

E) все-элементы вне тлавной диагонали равны единице

-

ฯ

-

2. Прямоугольная матрица $A$ размеров ' $m \times n$ называется ква

A) $m=n$ 甲

-

B) $m \neq n$ 甲

ฯ

-

C) $m<n$ -

D) $m>n$ ฯ

E) $m \leq n$ ฯ

-

3. При умножении матрицы на число умножаются..ๆ

А)все-элементы матрицы

Рисунок 4 - Тест до исправления.

1. Квадратная матрица называется диагональной, если...... А) все-элементы вне тлавной диагонали равны нулю-

В) все элементы побочной диагонали равны нулю-

C) все-элементы тлавной диагонали равны нулю-

D) все-элементы матрицы равны нулюণ

E) все-элементы вне-главной диагонали равны единице ศ

2. Прямоугольная матрица $A$ размеров ' $m \times n$ называется 'кв
A) $m=n$ ฯ
B) $\cdot m \neq n$ ฯ
C) $m<n$ ฯ
D) $m>n$ ฯ
E) $m \leq n$ ฯ
ศ

3. При умножении матрицы на -число умножаются..ๆ

А)все-элементы матрицы

В) все-элементы какого-нибудь-столбца

Рисунок 5 - Тест после автоматического исправления. 